\title{
Recent Trends in Collaborative, Open Source Drug Discovery
}

\author{
Anuradha Roy, Peter R. McDonald and Rathnam Chaguturu*
}

High Throughput Screening Laboratory, 2034 Becker's Drive, University of Kansas, Lawrence, KS66047, USA

\begin{abstract}
The pharmaceutical sector has traditionally played a predominant role in screening compounds against a molecular target and optimizing chemistry to develop drugs. The basic research studies in the academic sector have provided the pharmaceutical companies with many biological targets with potential impact on therapeutics. These welldefined contributions of the pharmaceutical and academic sectors in drug discovery field are being redefined to meet the fiscal and innovation challenges in the current drug discovery landscape. There is an increased capital and personnel investment in academia in the areas of highthroughput screening and technology transfer. The pharma has adopted an open innovation paradigm which seeks to complement internal intellect with external global talent and expertise, with the overall goal of expediting complex data interpretation and introduction of more effective and safe drugs. The pharmaacademia sectors are collaborating on several mutually beneficial alliances and the changing landscape at pharmaacademiainterface necessitates an urgent need for a better understanding of the role of academia in translational research and suitable technology transfer terms to sustain collaborative opportunities. The collaborative partnership between pharma and academia is expected to provide the required boost to identify novel targets and develop new and effective drugs.
\end{abstract}

Keywords: Drug discovery, HTS, Pharma, Academia, Open innovation, Probe discovery, High throughput screening.

\section{INTRODUCTION}

Pharmaceutical companies, faced with high R\&D costs [1] and dwindling product pipelines,are relying increasingly on academia for identifying therapeutically relevant drug targets to pursue, and hence have embarked on open innovation models in collaborating with academic institutions. Academic institutions, in turn, are leaning toward the pharmaceutical companies, non-profit organizations, philanthropies and the federal agencies such as NIH to seek funding for early and translational drug discovery research. Many such financial and innovation problems have ushered in a new era of pharma-academia collaborations for the advancement of drug discovery (Fig. 1). The collaborations are still evolving and require deeper recognition of the core missions and goals of the participants involved. A clear understanding is also required to examine and find ways to uncork this formidable drug discovery bottleneck. This has been pharma's predicament over the last several years. To ease this bottleneck, there is a need to identify all of the contributing factors which includes the drug targets being selected and pursued, finding tool/probe molecules against good therapeutic targets, and ways to transform these tool molecules to drug leads which are attractive for preclinical and clinical development.

The pharmaceutical companies have dominated drug discovery research over several years and many succeeded in marketing many blockbuster drugs in the last two decades [2]. In the current landscape, big pharma spends an average of 400 to 800 million dollars per new molecular entity

*Address correspondence to this author at the High Throughput Screening Laboratory, 2034 Becker's Drive, University of Kansas, Lawrence, KS66047, USA; Tel: (785) 864-1717; Fax: (785)864-1619;

E-mail: rathnam@ku.edu
(NME) [3], which is at least ten times more than that of the 1990s. An average of $30 \%$ of the marketed drugs recoups the return from $R \& D$ investment with the majority of drugs losing out on market competitiveness. Also, the patents for most of the marketed drugs, which previously compensated for the return on investment, are due to expire in the next few years [4] and are projected to challenge the bottom-line of most of the pharmaceutical companies. The increased regulatory demands for greater safety and efficacy, and increased post-marketing surveillance have also added to overall lower productivity. Project failures in pharma could be traced to lack of safety and efficacy. In addition to the well-known high attrition rates associated with ADME profiling, almost half of the project failures have been attributed to the therapeutic relevance of the biochemical targets that were being pursued. Pharma's underestimation of the significance of toxicobiology has also resulted in the selection of many suboptimal drug targets and hence the resultant lack of efficacy and safety.

The mission of academia has always been advancement of science through teaching and research.In recent years, there has been a shift in the guiding philosophy in the academic institutions when academia found itself in an environment of funding deficits. The core strength of academia lies in its pursuit of all known and rare aspects of biology and systems biology. This gives an edge to the academic scientists in identifying and validating novel molecular targets for various diseases, developing assays and to some extent, in probe discovery. In general, the mission of the industrial sector is not set up to do comprehensive basic research on biological targets, which warrants active collaboration between industry and academia [5].

To overcome the problems in the current landscape and to bridge the innovation gap, pharma needs better strategies 


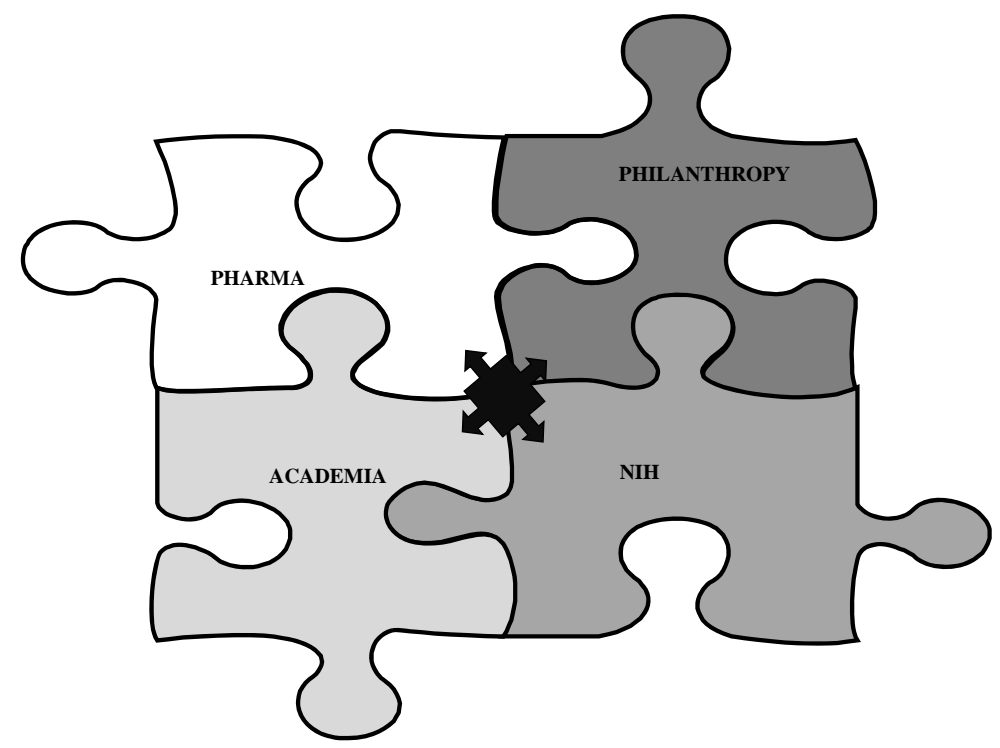

Fig. (1). Uncorking drug discovery bottleneck: a four-way partnership. New collaborative efforts between industry, academia/NIH and philanthropies are expected to overcome current challenges in drug discovery.

for identifying new and novel therapeutic targets, find molecules to probe new drug targets, and smart lead optimization strategies [6]. This has led to development of new business models and increased recognition of "Open Innovation" paradigm, a term initiallydescribed by Professor Henry Chesbrough [7], which now is interpreted as a concept promoting a collaborative and open engagement to introduce external ideas to complement internal ideas for enhancing a company's internal technology, and to expedite the path to market.In order to be more profitable and productive, the pharmaceutical industry has embraced an open innovation approach to share the drug discovery processes and data with academia [8]. In its simplest form, the academia contributes to target identification and disease validation research while the pharma steps in and makes the assay HTS ready, and carries on specialized screening campaigns [9]. The goal for a successful collaborative translational research is to ensure that the target is disease relevant, and from a toxicobiology perspective, the compounds against the target havegood safety and efficacy profiles.

\section{COLLABORATIVE DRUG DISCOVERY}

Target discovery and validation are two key steps essential for development of a relevant relationship between academia and pharma. Target validation is an essential factor to ensure that target correlation to disease is clear and that the target is druggable [10]. The target selection in pharmaceutical sector is driven by the return on investment and increasing shareholder value. The pharmaceutical industry has operated mainly in the low risk territory since diseases afflicting large patient populations bring in more revenue for industry and are preferred over rare disorders affecting small population groups which predict smaller revenues. Academia, by the nature of its mission, has worked in this unchartered territory of high risk and low reward, but for it to make head way, it is limited by the availability of easily adaptable technology formats to manipulate the highly refractory targets [11]. There are about 7,000 diseases that afflict mankind, but treatments are available for less than $3 \%$ of these diseases despite the fact that genetic basis for most of these diseases is known [12]. As it stands, there are about 25,500 protein coding genes in the human genome, and $\sim 10^{60}$ possible small molecules with 30 or less heavy atoms [13]. Of a large number of potential targets in human genome, the FDA approved drugs target less than $0.5 \%$ of the entire human genome, with 289 drugs targeting just 133 genes. This indicates that there is plenty of opportunity to characterize complex biology of known and less characterized targets that may have implications in the treatment of cancers, heart disease and other diseases which exhibit genetic complexity and are influenced by environmental factors. Many such previously ignored targets will emerge into focus as potential therapeutic targets with increased academic input in target identification and validation in the new business models being pursued by pharmaceutical companies. In addition to new target research, collaborations between the public-private sectors also include characterization and identification of ADME/tox properties of many drug candidates at early stages of drug discovery. The eTOX program of EU's Innovative Medicines Initiative (IMI, http://www.imi.europa.eu), facilitates data sharing between the big pharma and academia. The IMI's goals of creating quality toxicological database with high quality in vivo and in vitro data and predictive toxicological models will have the potential to impact the high attrition rates associated with advanced drug discovery programs.

\section{PROBE DISCOVERY IN THE PUBLIC DOMAIN}

One of the immediate goals of academic research in drug discovery is to identify tool molecules/agents like small molecules, new chemistries, antibodies, or biomarkersthat can be used to study a specific pathway or biological process or event. The term 'probe' broadly encompasses all types of tool molecules and was recently endorsed and promoted by the NIH Roadmap Initiative to highlight compounds which are reasonably specific at early stages of compound screening against a molecular target. An ideal probe is a 
small molecule that is novel and drug like with submicromolar affinity and selective to the therapeutic target, and has the potential to be the starting point for lead optimization studies. Alternatively, the probe may be used to study challenging refractory biology [14]. Other desirable aspects of a new probe include a proven chemical identity with a defined structure, a clear and quantifiable structureactivity relationship over a 2-3 log drug concentration range, identification of more than one chemotype with an analogous activity profile and a proven efficacy and potency around 3 orders of magnitude.

The changing landscape between academia and pharma, and an unprecedented investment by NIH in creating and fostering a Roadmap Initiative has resulted in emergence of probe discovery in academia [15]. In addition, academia, previously averse to high throughput screening, now fully embraces high throughput screening of diverse compound libraries as a necessity, and as a fully recognized discipline. This is reflected in numerous high throughput screening (HTS) centers that are harbored in universities all over the U.S. and provide diverse aspects of screening services to investigators [16]. This change in academic philosophy offers great potential for coming up with new therapeutic targets and new modalities.

The establishment of HTS centers in academia correlates with the commercial availability of chemical libraries. There are now over 20 major vendors, offering a total of almost 11 million compounds [17]. In addition to diverse and focused commercial libraries, many academic centers like the High throughput screening laboratory at the University of Kansasare privy to institutional chemistries as part of the chemical library holdings. The academic institutions have also invested heavily in new and sensitive cutting edge technology to equip their high throughput screening centers. In addition to the technology, some academic centers also provide exceptional medicinal \& computational chemistry support, which is critical in the evolution of a screening 'active' into an ideal drug.

\section{Major Probe Discovery Programs in Public Domain: NIH Roadmap Initiative and the EU-OPENSCREEN}

$\mathrm{NIH}$ through its Roadmap initiative set up a Molecular Libraries Program (MLP) to help mine human genome and to explore new ways to study the functions of genes and signaling pathways. MLPCN, Molecular Libraries Probe Production Centers Network, as part of the MLP, provides academic researchers with an opportunity to perform largescale compound screening for identification of small molecules that can be optimized as probes. The MLPCN, a nationwide consortium of small molecule screening centers, is an unprecedented investment supporting technology development involving instrumentation, assay development, chemical diversity, ADMET, high throughput screening and chemoinformatics. The program started as a pilot phase in the year 2005, developing a network of 10 screening centers with a major emphasis on bioassays. It is now in its production phase, with an emphasis on chemical probes. The researchers from US and other countries can access NIH's MLPCN by submitting grants for assay development (R21) or for high throughput screening (R03, Fast Track) for screening assay ready targets at MLPCN against $\sim 350,000$ compounds in uHTS mode at no cost, and with chemistry support. The screening centers established by NIH as well as the academic high throughput screening centers have recently allowed the academic researchers working on a wide variety of targets to submit their assays and facilitate screening campaigns. The NIH roadmap funded biochemical, cellular and model organism based assays for any biological target for screening their compound libraries. The actives from such screens could function as probes, or tools to further define target/cellular relevant to physiology and disease or serve as starting points for hit to lead development for drug discovery. The current emphasis of NIH drug discovery is to promote probe/ drug development for rare and neglected diseases (TRND program, http://nctt.nih.gov/trnd).

EU-OPENSCREEN, a European counterpart of the NIH Roadmap initiative, is an association of HTS centers across Europe which offers resources for executing high throughput screening. The EU-OPENSCREEN supports European academic hit discovery and optimization, and provides informatics support and a publicly accessible databasefor providing screening results, assay protocols and chemical information. It is the most ambitious open access high throughput drug discovery platform in the public domain involving 19 academic and non-profit institutions from 17 European countries, and brings together biomedical scientists representing all related and required disciplines. The EU- OPENSCREEN provides European researchers access to the most advanced screening technologies and has a well-established framework for IP management.

In addition to the above programs, others significant collaborative initiatives require special mention. The IGC (International Genomics Consortium, http://www.intgen. org), a non-profit medical research organization, is focused on diagnostics, treatment and prevention of cancer and complex diseases by molecular profiling, characterization of differential biomarker expression for personalized medicine. The IGC program includes the National Institutes of Health's The Cancer Genome Atlas project, TCGA, which generates genomic and clinical data for specific cancer types for use by the cancer research community.

\section{OPEN-INNOVATION STRATEGIES}

As shown in Table 1, the origins of a number of FDA approved drugs can be traced to collaborative efforts between pharma and academia/NIH. In recent years, the pharmaceutical companies have established new R\&D structures to foster open-innovation dialogue with academia [18]. The R\&D centers of excellence have been set up at many academic institutions and new research initiativesfor expanding the systems biology database that are available for public mining (Table 2). The collaborations range from exploring basic biology of targets of therapeutic interest, novel target identification to collaborations on replacement stem cell therapies. A few representative collaborations are highlighted as follows:

Eli Lilly-PD2 Initiative (https://pd2.lilly.com): In this initiative, Eli Lilly is seeking to test the therapeutic potential of novel compounds synthesized in university/biotechnology laboratories in its diseaserelevant phenotypicdrug discovery platform. 
Table 1. Representative Drugs Originating from Pharma-Academia-NIH Collaborations

\begin{tabular}{|c|c|c|c|}
\hline Drug & Type & Academia/NIH & Pharma \\
\hline Altima & Antifolic acid agent for oncology & Princeton University & Eli-Lilly \\
\hline DOXIL & Doxorubicin liposome for ovarian cancer & Hebrew Univ, Hadassah Hospital & $\mathrm{J} \& \mathrm{~J}$ \\
\hline ELMIRON & Glycosaminoglycan for bladder pain & Univ of California & $\mathrm{J} \& \mathrm{~J}$ \\
\hline Gardasil & HPV vaccine for cervical cancer & KU, National Cancer Inst & Merck \\
\hline Kepivance & Keratinocyte GF for oral mucositis & National Cancer Inst, NIH & Amgen \\
\hline LEUSTATIN & Antineoplastic agent for hairy cell leukemia & Scripps, Brigham Young & $\mathrm{J} \& \mathrm{~J}$ \\
\hline Myozyme & Recomb alpha-glucosidase for Pompe disease & Erasmus Univ Medical Ctr & Genzyme \\
\hline Nizoral & Ketoconazole for dandruff treatment & Univ of Tennessee & $\mathrm{J} \& \mathrm{~J}$ \\
\hline Pepcid & Combination antacid \& $\mathrm{H} 2$ antagonists for heartburn & Brigham and Women's Hospital & $\mathrm{J} \& \mathrm{~J} / \mathrm{Merck}$ \\
\hline Prezista (TMC114) & Protease inhibitor for HIV & Univ of Illinois & $\mathrm{J} \& \mathrm{~J}$ \\
\hline $\begin{array}{l}\text { PROCRIT }^{\circledast} / \text { EPREX }^{\circledR}: \\
\text { epoetin alfa }\end{array}$ & Anemia & Univ of Chicago & $\mathrm{J} \& \mathrm{~J}$ \\
\hline $\begin{array}{c}\text { REMICADE }^{\circledR} \text { : anti-TNF } \\
\text { mAb }\end{array}$ & Immune disorders & NYU & $\mathrm{J} \& \mathrm{~J}$ \\
\hline $\begin{array}{l}\text { ReoPro }^{\circledR} \text { : GPIIb/IIIa } \\
\quad \text { receptor mAb }\end{array}$ & Cardiac ischemia & SUNY & $\begin{array}{c}\text { Lilly \& Centocor, } \\
\text { J\&J }\end{array}$ \\
\hline YONDELIS & Marine-derived anti-tumoral agent & Univ of Illinois & $\mathrm{J} \& \mathrm{~J}$ \\
\hline Zolina & Histone deacetylase inhibitor for oncology & Columbia, Sloan Kettering, & $\begin{array}{l}\text { Aton Pharma \& } \\
\text { Merck }\end{array}$ \\
\hline
\end{tabular}

The selected molecules and promising compounds originating from academia are tested in the Eli Lilly phenotypic discovery platform and the profiling data and the secondary assay information is shared with the academic researchers. This provides an opportunity for academic researchers to test their previously uncharacterized compounds into Lilly's drug discovery and development process. All compounds with promising biological activity are identified and future collaborations are established for furthering drug discovery efforts.

(2) Merck-Sage Bionetworks: SAGE is an open access, non-profit organization established by Merck (http://sagebase.org). Merck has deposited data from human and mouse disease models data from the Rosetta platform. This provides the public with a database on systems biology network and also the required downloadable computational disease biology software tools for data access and analysis. The longterm goal of network biology is aimed at designing better and more targeted drugs based on systems network models.

(3) GSK-caBIG Collaboration: GSK released genomic profiling data for over 300 cell lines via the National Cancer Institute's Cancer Bioinformatics Grid ${ }^{\mathrm{TM}}$ (caBIG), a network of infrastructure and tools that enables the collection, analysis, and sharing of data. The site provides valuable information for genomic profiles for a wide variety of tumors, including breast, prostate, lung and ovarian cancers. The public access allows any researcher to download the open-source GSK cancer data through caArray. The GSK has also made available in public domain its data for over 13,500 compounds confirmed to inhibit malaria 
Table 2. Partnerships Between Pharma and Academia

\begin{tabular}{|c|c|c|}
\hline Private Sector & Public Sector & Drug Target Research Area \\
\hline Glaxo Smith Kline & Harvard Immune Disease Institute & Immuno-inflammatory drug discovery; $\$ 25$ million/3years \\
\hline Glaxo Smith Kline & Harvard Stem Cell Institute & $\begin{array}{l}\text { Heart disease and cancer } \\
\$ 25 \text { million/5years }\end{array}$ \\
\hline Astra Zeneca & Columbia & Metabolic diseases; \$Multimillion \\
\hline Pfizer & University of Pennsylvania & $\begin{array}{l}\text { Scientific research, clinical development and clinical care and policy; } \$ 15 \\
\text { million }\end{array}$ \\
\hline Pfizer, Entelos & $\begin{array}{l}\text { University of California, MIT, } \\
\text { University of Massachusetts }\end{array}$ & $\begin{array}{l}\text { Regulation of energy metabolism expand the understanding diabetes and } \\
\text { obesity pathobiology; \$14 million/ } 3 \text { years }\end{array}$ \\
\hline Johnson \& Johnson & Academic universities & Two partnership-focused units to nurture early academic research \\
\hline $\begin{array}{l}\text { GlaxoSmithKline, Pfizer Inc. and } \\
\text { AstraZeneca }\end{array}$ & $\begin{array}{l}\text { Critical Path Institute, a nonprofit } \\
\text { partnership with the FDA }\end{array}$ & $\begin{array}{l}\text { Coalition Against Major Diseases to share data on thousands of } \\
\text { Alzheimer's patients in hopes that the extra information will spark new } \\
\text { ideas for treatments }\end{array}$ \\
\hline Eli Lilly & $\begin{array}{l}\text { Open-access submission of } \\
\text { compounds }\end{array}$ & Phenotypic Drug Discovery platform \\
\hline GSK & Open access database & $\begin{array}{l}\text { Genomic \& protein expression profiling data for over } 300 \text { cancer cell } \\
\text { lines via the NCI"s cancer Bioinformatics Grid }{ }^{\mathrm{TM}} \text { for academia to mine }\end{array}$ \\
\hline Merck-SAGE & Open access database & $\begin{array}{l}\text { sharing and disseminating complex data representing disease biology } \\
\text { [genetic (SNP, copy number variations), RNA expression (mRNA, } \\
\text { miRNA, other non-coding RNA) }\end{array}$ \\
\hline Pharma Consortium & $\begin{array}{l}\text { CTSA Pharmaceutical Assets Portal- } \\
\text { NIH }\end{array}$ & $\begin{array}{l}\text { To improve information exchange regarding drugs available for } \\
\text { repurposing-proactively engage pharma in data sharing }\end{array}$ \\
\hline GSK & Emory Institute for Drug Discovery & Drug discovery for rare diseases like malaria \\
\hline
\end{tabular}

List of some recent collaborations between pharma and academia in specific drug target areas.

parasite growth, which are useful tools that academicians can employ for investigating functional mechanisms.

The SGC (Structural Genomics Consortium) is an open-access, not-for-profit, public-private partnership to contribute to basic biology relevant to drug discovery using large-scale 3D structures of proteins of therapeutic importance from humans and their parasites. The SGC includes collaborations between the Universities of Toronto and Oxford and the KarolinskaInstitutet in Stockholm with GSK, Eli Lilly, Pfizer, the Novartis Research Foundation, the Wellcome Trust, and Canadian agencies. The SGC has also collaborated with the Chemistry and biochemistry departments of University of Oxford, the NIH Chemical Genomics center in Washington, and GlaxoSmithKline (GSK) to set up open-access chemical probes for epigenetic proteins.

(5) The University College London (UCL) established collaborationsto advance its expertise on stem cell replacement therapies. One of the collaborations was established between University College London (UCL) and Astrazeneca for the treatment of diabetic retinopathy. Another collaboration between UCL and Pfizer aims to utilize Pfizer's expertise in drug design and delivery to advance the work of UCL researchers in the field of stem cell-based therapies for agerelated macular degeneration (AMD).

The open source approach, in which major pharmaceutical companies allow public access to the information previously held confidential, is highly beneficial to pharma who now employs global intellect, at no cost, in dissecting large volumes of data. Using this as a new business model, the pharma hopes to accelerate drug discovery by improving research efficiency and output. On the same note, the academic community, which normally cannot undertake large, costly drug, biomarker and molecular screening campaigns leading to probe development, can further their research using the enormous datasets deposited in the public domain by large pharma.

\section{COST EFFECTIVE DRUG REPURPOSING}

The concept thata single gene target corresponds to a single disease has been replaced by the concept that a target is part of a complex signaling network; the gene expression profiles of BRCA 1 and 2, popularly associated with breast cancer,have been shown to be associated with prostate cancer and ovarian cancer as well. Likewise, most of the approved drugs exhibit pleiotropic effects and can act at known and unknown sites resulting in 'on' and 'off' target effects. There is an increased emphasis on a systems biology approach in pharma to understand each therapeutic target in relation to its nearest neighbors in the metabolic pathway 
Table 3. Avenues for Partnerships Between Academia and Industry

\begin{tabular}{|c|c|c|c|}
\hline Parameter & Grant & Contract & Collaboration \\
\hline \hline Research idea originated by & PI & Pharma & Pharma \& PI \\
\hline Research plan developed by & PI & Pharma & Pharma \\
\hline Project Costs covered by & Pharma & Pharma & Pequires Pharma's input \\
\hline Results published & PI's discretion & Requires Pharma's approval & Pharma \& PI \\
\hline IP and data ownership & PI & Pharma refusal \\
\hline Project direction & PI & Pharma & Pharma \\
\hline
\end{tabular}

The origin of the project defines the course of partnership between academia and pharma. A grant supports an investigator's research without any encumbrances, while a contract establishes a fee for service project aimed at addressing a defined problem. Collaboration is a true joint venture with management by all partners.

involved. The realization of the importance of network and systems biology has led to a renewed interest in drug repurposing or drug repositioning.A major focus for drug repurposing, which benefits big pharma, is to re-pursue drug candidates that did not succeed in advanced clinical trials, for reasons other than safety, for potential new therapeutic applications. The pharma can clearly benefit commercially from finding new indications for previously failed advanced stage drug candidates, around which the patent space can be easily exploited. The profitability from repurposing a previously approved drug is far more complex and will depend on the operational space around previously published patents, which includes public information on new indication value and any anticipated the new use of a known drug.

Pharma is now taking advantage of this promiscuity to revive its dwindling pipelines and go after repurposing the drugs that have the potential to be marketed for new therapeutic indications. Drug repurposing or finding new uses for marketed or pipeline drugs is extremely costeffective as these compounds have the necessary safety profile, and further investigation requires identification of new therapeutic indications. This also points to the fact that many of the approved compounds are not highly target specific, which results in many side-effects and also explains why most compounds fail during the Phase II clinical trial stage. Based on the CTSA Pharmaceutical Assets Portal drug repurposing data, in the last 60 years, $\sim 13,800$ compounds reached Phase II \& III clinical trials, but only 2,800 compounds were approved by FDA. The subsets of 13,800 compounds, which are not associated with safety issues, are good candidates for drug repurposing. One of the goals of CTSA is to facilitate interactions between matched academic investigators and the pharmaceutical industry to develop research partnerships based on compounds shelved at the clinical stage.

One of the goals of CTSA is to facilitate interactions between matched academic investigators and the pharmaceutical industry to develop research partnerships based on compounds shelved at the clinical stage. The CTSA (http://www.ctsaweb.org) represents pharma-academia integration efforts which involve matching interests of around 348 researchers with diverse disease interests from 45 universities and 8 pharmaceutical companies (Pfizer, Merck, GSK, Novartis, Genentech, Abbott, Eli Lilly, and AstraZeneca).

\section{AVENUES FOR PARTNERSHIPS BETWEEN ACADEMIA AND PHARMA}

A successful collaboration has an overall goal to produce good science and generate valuable intellectual property that supports innovation by private sector partners. The recent business models are geared towards corporations expressing interest in co-development, risk/share and collaborative research agreements with options. A successful academia and pharma partnership requires complete understanding and respect for the guiding principles of institutional interests and bottom-lines. Problems develop due to divergent interests, work cultures, timelines and poor project management. The academic drawbacks lie in seeking compensation for indirect costs, poor negotiation skills and partial/incomplete understanding of legal concepts, intellectual property (IP), contract law, Federal and state regulations, and lack of knowledge of policy positions. Industry has to ease its restrictions on timely publication, research growth, grant support for ongoing academic financial needs, rigid IP policies and ownership of results and exclusivity. The academic-pharma alliances may be financially mediated via 1) a grant, which supports an investigator's research without any encumbrances, 2) a fee for service contract for solving a defined problem or 3) a true collaboration with management by all partners (Table $\mathbf{3}$ ).

\section{Academia and Pharma Collaborations: Anopportunity Toadvance the Frontiers of Drug Discovery Research and Promote Technology Innovation}

Together, the pharma and academia have a unique opportunity to create an enduring partnership that will accelerate the discovery of innovative medicines. In this relationship, the academia receives more research dollars and engages in more innovative and applied research. Academic faculty and students, through alliances with pharmaceutical companies, also have an opportunity to be trained in timeline based targeted research and be involved directly in drug discovery and development. Academic researchers learn to recognize how their projects and areas of research can progress along new drug discovery approaches and alliances with the pharma could help accelerate development of projects that would otherwise languish within the walls of their laboratories. University technology transfer offices provide academia with a means to secure product development and circumvent traditional licensing outcomes. Since all collaborations are not the same, the university 
technology transfer offices deal with each academia- pharma collaboration on case-by case basis andwork to resolve the complexities involved in handling academic interests vis-àvis their corporate collaborators.

On the other side of the relationship, the pharma is exposed to a greater understanding of toxicobiology and systems biology, receives information on new and more relevant therapeutic targets to pursue for drug discovery. Pharmaceutical companies can use the basic information from academia about new molecular probes which could serve as starting points for 'hit to lead' development and help maintain their project pipelines. The training provided to university students through such alliances will help feed employment searches for targeted/trained hires.

For partnerships to be successful, both sides must identify definitive deliverables in accordance with their core missions: the pharma shares the intellectual property rights with the academia but can have a greater say in licensing with the option for a first right of refusal. Academia should agree with pharma in providing opportunities for 'product' development and the pharma should readily support the academia's need for timely research publications and data for seeking additional grant support.

\section{SUMMARY}

The long and difficult process of drug discovery coupled with dwindling pipelines and low revenues have increased risks for the pharmaceutical sector. Pharmaceutical industry is at cross-roads with regards to what to do next in reinventing itself to be more profitable and in discovering new products. For revitalizing drug discovery, there is a new found realization on the part of the pharmaceutical industry for its need to spread the financial and intellectual burden of early stage drug discovery research. By adopting open innovation, the pharmaceutical companies can capitalize on external and internal information to be more creative, and productive, and hence more profitable. In addition to open innovation, pharma is refocusing on academia for pursuing basic biology and validating new disease-relevant targets. Academia, while pursuing the new targets needs industry input to process the targets and render them more amenable for late drug discovery. Thus, in the new landscape of drug discovery, there is increased interdependence of both academia and pharma. Current trends in the areas of drug discovery and healthcare support the role of academia in easing the drug discovery bottleneck, and in solving the unmet medical needs. It has become clear that pharma, disease foundations and academia working togetherfor common good will revitalize innovationand help introduce novel and safer drugs.

\section{ACKNOWLEDGEMENTS}

KU-HTSL is a KU Cancer Center Shared Resource, and is funded In part by NIH/NCRR, COBRE grant P20 RR015563.

\section{CONFLICT OF INTEREST}

None declared.

\section{REFERENCES}

[1] Vernon, J.A.; Golec, J.H.; Dimasi, J.A. Drug development costs when financial risk is measured using the Fama-French three-factor model. Health Econ., 2010, 19 (8), 1002-1005.

[2] Zycher, B.; DiMasi, J.A.; Milne, C.P. Private sector contributions to pharmaceutical science: thirty-five summary case histories. Am. J. Ther., 2010, 17 (1), 101-120.

[3] DiMasi, J.A.; Hansen, R.W.; Grabowski, H.G. The price of innovation: new estimates of drug development costs. J. Health Econ., 2003, 22 (2), 151-185.

[4] The patent cliff steepens. Nat. Rev. Drug Discov., 2011, 10 (1), 1213.

[5] Frye, S.; JanZen, B. Discovering Academia www.FuturePharma US.com[Online], 2007, p. 1-4.

[6] Wagner, J.A. Open-minded to open innovation and precompetitive collaboration. Clin. Pharmacol. Ther., 2010, 87(5), 511-515.

[7] Chesbrough, H. Open innovation: The new imperative for creating and profiting from technology. Harvard Business School Press: Harvard, MA, 2003.

[8] Tralau-Stewart, C.J.; Wyatt, C.A.; Kleyn, D.E.; Ayad, A. Drug discovery: new models for industry-academic partnerships. Drug Discov. Today, 2009, 14 (1-2), 95-101.

[9] Paul, S.M.; Mytelka, D.S.; Dunwiddie, C.T.; Persinger, C.C.; Munos, B.H.; Lindborg, S.R.; Schacht, A.L. How to improve R\&D productivity: the pharmaceutical industry's grand challenge. Nat. Rev. Drug Discov., 2010, 9 (3), 203-214.

[10] Lindsay, M.A. Target discovery. Nat. Rev. Drug Discov., 2003, 2 (10), 831-838.

[11] Wellman-Labadie, O.; Zhou, Y. The us orphan drug act: rare disease research stimulator or commercial opportunity? Health Policy., 2009, 24, 24.

[12] Overington, J.P.; Al-Lazikani, B.; Hopkins, A.L. How many drug targets are there? Nat. Rev. Drug Discov., 2006, 5 (12), 993-996.

[13] Hert, J.; Irwin, J.J.; Laggner, C.; Keiser, M.J.; Shoichet, B.K. Quantifying biogenic bias in screening libraries. Nat. Chem. Biol., 2009, 5 (7), 479-483.

[14] Roy, A.; McDonald, P.R.; Sittampalam, S.; Chaguturu, R. Open access high throughput drug discovery in the public domain: a mount everest in the making. Curr. Pharm. Biotechnol., 2010, 11 (7), 764-778.

[15] Austin, C.P.; Brady, L.S.; Insel, T.R.; Collins, F.S. NIH molecular libraries initiative. Science, 2004, 306 (5699), 1138-1139.

[16] Gordon, E. J. Small-molecule screening: it takes a village. ACS Chem. Biol., 2007, 2 (1), 9-16.

[17] Roy, A.; Taylor, B.; McDonald, P.; Price, A.; Chaguturu, R. Hit-toprobe-to-lead optimization strategies: a biology perspective to conquer the valley of death. In Handbook of Drug Screening, Seethala, R.; Zang, L., Eds. Informa Healthcare: 2009; pp. 21-55.

[18] Hunter, J.; Stephens, S. Is open innovation the way forward for big pharma? Nat. Rev. Drug Discov., 2010, 9, 87-88. 